\title{
Mise en place d'une collection d'abeilles (Hymenoptera, Apoidea) dans le cadre d'une étude de la biodiversité
}

\author{
Par Hugues MOURET *, Gabriel CARRE **, Stuart PM ROBERTS ***, Nicolas MORISON ** et \\ Bernard E VAISSIERE **
}

\begin{abstract}
In the framework of the European ALARM project, we used sampling methods to assess pollinator diversity (bees and syrphid flies) and abondance, and therefore started reference collections. Identification required killing, pinning, and adequate preparation of the insects sampled. We present a set of guidelines for the preparation of bee specimens and a protocol for processing specimens caught in liquid media. We also provide recommendations to fill out labels for collection purposes. Finally, we suggest some techniques that can help keeping a well-curated bee collection.
\end{abstract}

Résumé. Dans le cadre de nos travaux sur la biodiversité des pollinisateurs au sein du programme européen ALARM, nous présentons quelques conseils pour constituer une collection d'Apoïdes. La détermination des espèces d'abeilles passe par la nécessité de tuer, d'épingler et d'étaler les insectes. Ainsi, une préparation adaptée des spécimens facilite grandement le travail de détermination. Nous présentons donc un protocole de préparation d'abeilles collectées dans des pièges à liquide. Nous exposons également quelques règles d'étiquetage des abeilles. De plus, nous abordons les problèmes de pérennité d'une collection de référence ainsi que les conditions de conservation.

Mots-clés. Biodiversité, collection entomologique, abeilles, techniques de préparation, identification.

Appréhender la biodiversité des abeilles

\section{Contexte de l'étude : une volonté européenne}

Les conséquences du déclin de la biodiversité sur les écosystèmes sont de plus en plus perceptibles (Stuart Chapin et al. 2000) alors même que les conséquences positives de la biodiversité sur les activités humaines commencent à être reconnues et même chiffrées en termes économiques (Costanza et al. 1997). Face à cette situation, le programme européen ALARM (Assessing LArge-scale environmental Risks for biodiversity with tested Methods; http://www.alarmproject.net; Settele et al. 2005) a pour objectif sur cinq ans (2004-2008) d'évaluer les risques encourus par la biodiversité terrestre et aquatique et l'impact potentiel de son déclin à l'échelle de l'Europe. ALARM constitue à ce jour le plus important programme de recherche sur la biodiversité financé par l'Union Européenne. Il comprend 4 modules - changements climatiques, produits chimiques, espèces invasives et pollinisateurs complétés par un module transversal socioéconomique.

\footnotetext{
* Arthropologia, rue des roches, F-69210 Sourcieux les mines, France. E-mail : hmouret@arthropologia.org

** Laboratoire de Pollinisation Entomophile UMR 406, Ecologie des Invertébrés, INRA Site Agroparc, F-84914 Avignon Cedex 9, France. E-mails: carre@avignon.inra.fr, morison@avignon.fr vaissier@avignon.inra.fr.

*** Centre for Agri-Environmental Research, School of Agriculture, Policy and Development, University of Reading, Earley Gate, PO Box 237, Reading RG6 6AR, United Kingdom.

E-mail: s.p.m.roberts@reading.ac.uk
}

La survie et l'évolution de plus de $80 \%$ des espèces végétales et la production de $84 \%$ des espèces cultivées en Europe dépendent directement de la pollinisation par les insectes et, plus particulièrement, par les abeilles dont il existe près d'un millier d'espèces en France (Williams 1994; Buchmann \& Nabhan 1996). La pollinisation des cultures entomophiles constitue un service économique bien visible, mais fragile, qui dépend pour une large part de l'agro-écosystème qui entoure les parcelles (Kremen et al. 2002, 2004). Dans le cadre d'ALARM, les 11 partenaires du module Pollinisateur ont pour objectif de déterminer l'évolution récente des populations de pollinisateurs en fonction des risques identifiés et mesurés, comme la dégradation des habitats naturels, et l'impact de cette évolution.

Pour la première année du programme ALARM, nous avons testé différentes méthodes pour évaluer l'abondance et la diversité des pollinisateurs selon un protocole commun dans plusieurs pays européens (Allemagne, France, Grèce, Pologne, Royaume-Uni et Suède). La seconde étape a été d'évaluer l'impact potentiel du déclin des populations de pollinisateurs sur les cultures et la flore sauvage.

Pour la France, l'expérimentation a été conduite par l'INRA d'Avignon (Laboratoire de Pollinisation Entomophile) et a pris en charge l'évaluation de l'impact de ce déclin sur les cultures. Sur les deux années d'expérimentation, 5905 spécimens répartis sur les 6 familles d'abeilles (Rasmont et al. 1995; Michener 2000) ont été capturés selon deux méthodes principales 


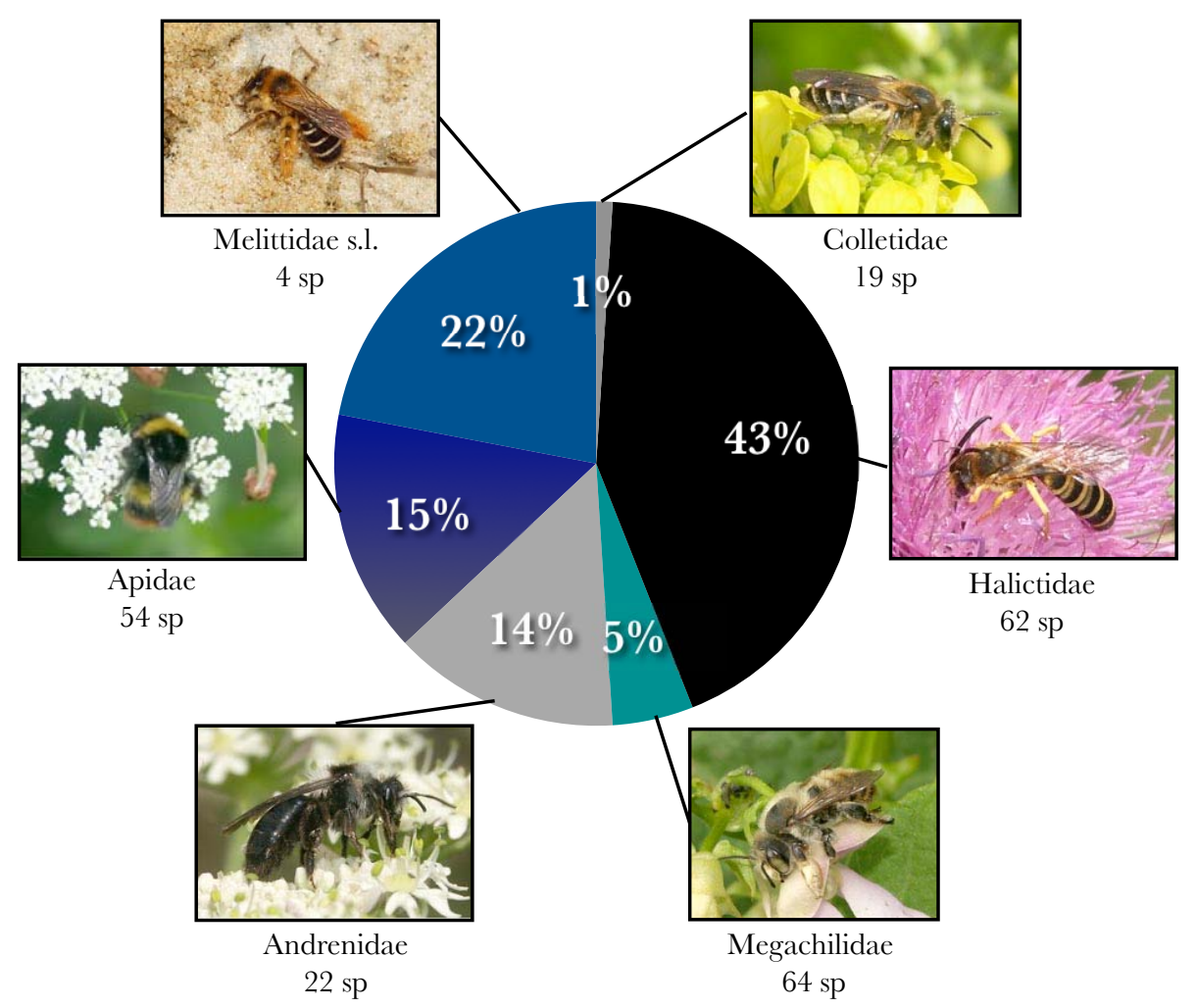

Figure 1. Abondance et diversité des abeilles capturées dans le sud-est de la France de mai à août 2004 \& 2005 dans le cadre du programme ALARM . Pour chaque famille d'abeilles, l'abondance relative est indiquée en \% (avec un effectif total de 5905 spécimens) et le nombre d'espèces échantillonnées accompagne le nom des familles.

de relevés que sont les captures au filet et par coupelles colorées (Figure 1). La mise en collection de ces spécimens a nécessité la mise en pratique de règles concernant la préparation, l'épinglage, l'étalage et l'étiquetage. Voici donc une synthèse illustrée qui fournira, nous l'espérons, une première base pour se lancer dans la réalisation d'une collection de référence.

\section{Une collection entomologique, pour quoi faire?}

Quelles sont aujourd'hui les motivations pour constituer une collection entomologique? Pourquoi ne pas s'en tenir aux observations, comme cela peut être le cas dans plusieurs disciplines naturalistes (ornithologie, herpétologie...) ? La France compte près de 40000 espèces d'insectes, soit presque 10 fois plus que les mammifères sur la planète ! Du fait de leur taille souvent réduite, certains caractères diagnostiques ne peuvent être observés que sous une loupe binoculaire. La détermination des insectes nécessite en conséquence très souvent la mise à mort et la préparation.

Quelques entomologistes expérimentés peuvent déterminer un faible nombre d'espèces sans en passer par la mise en collection. Toutefois, il s'agit d'une minorité d'espèces que seule la spécialisation et l'expérience permettent de mieux connaître. Notons, que cette spécialisation passe en général par la constitution de collections de références.

$\mathrm{Au}$ moment de constituer une collection entomologique, il est important de se poser la question de sa pérennité. Il est nécessaire, pour l'intérêt scientifique d'une collection de soigner la préparation des spécimens, de constituer un étiquetage réfléchi, complet et pertinent.

Nous présentons, ci-après des protocoles pour préparer les spécimens conservés dans l'éthanol, pour effectuer le montage et l'étiquetage, ainsi que plusieurs conseils pour la conservation optimale d'une collection.

\section{Préparation d'abeilles conservées dans l'alcool}

Lorsque l'on prélève beaucoup d'insectes, par exemple lors de piégeages de masse, il n'est généralement pas possible de tous les préparer au jour le jour. De plus ce type de récoltes est souvent réalisé à l'aide coupelles colorées, par attraction des insectes dans un liquide (eau + détergent) ; aussi les spécimens capturés sont conservés dans de l'alcool (en général une solution aqueuse d'éthanol)

À la sortie des flacons d'alcool, les insectes sont souvent cassants et présentent une forte dégradation de l'aspect général de la pilosité. Il est donc nécessaire de les préparer pour leur redonner un aspect plus naturel qui facilitera leur détermination ultérieure. On décrit ici le protocole de T. Griswold (USDA Bee Lab., Logan, Utah - http://online.sfsu.edu/ beeplot/), qui s'est avéré très utile et performant au vu de nos besoins (Figure 2).

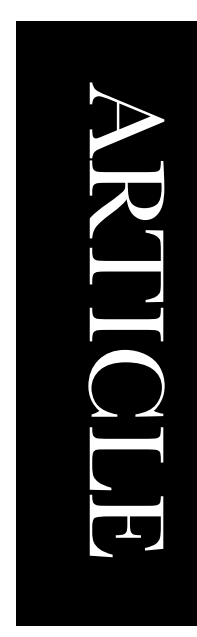


Protocole de préparation (Figure 2):

1- Récupérer le contenu du flacon dans une passoire à maille fine (pour éviter de perdre les abeilles de taille inférieure à $2 \mathrm{~mm}$ ) et rincer à l'aide d'une pissette d'eau distillée;

2- Puis verser le contenu de la passoire dans un récipient avec de l'eau tiède $\left(30^{\circ} \mathrm{G}\right.$ environ) et quelques gouttes de liquide vaisselle; agiter pendant une minute (par exemple à l'aide d'un agitateur magnétique);

3- Reverser ensuite le contenu dans la passoire et rincer sous un filet d'eau en ayant soin de protéger les échantillons avec la main;

4- Egoutter la passoire et essuyer l'excès d'eau avec un torchon;

5- Rincer avec de l'éthanol $96 \%$;

6- Placer ensuite les insectes dans du papier absorbant et remuer les spécimens pour un premier séchage; changer le papier jusqu'à absorption quasi totale du liquide;

7- Faire une poche avec une serviette en papier propre et agiter les insectes énergiquement (mais précautionneusement) pendant 20 secondes environ;

8- Un brossage, à l'aide d'un pinceau, permet de peigner et de séparer les poils agglutinés. Il n'est pas inutile de "peigner" les abeilles très poilues sous un sèche-cheveux, afin de leur redonner un aspect plus "naturel"; notamment Dasypoda, Bombus, Anthophora, etc. ;

9- Monter les insectes sur épingle (ou minutie pour les plus petites espèces); éventuellement exposer les genitalia des mâles. On peut ensuite les placer à $60 \mathrm{~cm}$ environ d'un petit ventilateur à air chaud (1500 W) pendant 6h-8h pour mieux les sécher, faciliter l'évaporation de l'alcool et de l'eau et ainsi éviter que les poils ne restent collés. Les durées sont à titre indicatif et il n'est pas dommageable de les laisser plus longtemps.

\section{Remarques:}

Un étiquetage rigoureux est indispensable dès que les spécimens sont sortis des récipients de collecte annotés, pour éviter toute perte de données.

Parfois lors de l'épinglage, une goutte de liquide ressort ce qui a tendance à ré humidifier les poils ; on peut alors tamponner légèrement avec un papier absorbant et passer de nouveau le pinceau, voire le sèche-cheveux.

Si les abeilles ont été stockées dans des solutions d'alcool plus concentrées ou que les spécimens restent cassants. Il est alors nécessaire de les "relaxer" afin d'éliminer l'excès d'alcool. Pour cela, on peut replacer les insectes rincés dans des flacons d'eau distillée. Ce trempage permet de baigner convenablement les tissus et donc de diluer grandement l'alcool qui imprègne le milieu intérieur. La durée de trempage dépend de la taille de l'insecte, de la concentration de la solution d'alcool de stockage au départ et de la durée (en jours) pendant laquelle le spécimen a été conservé dans cette solution : il faut laisser les spécimens suffisamment pour que les insectes redeviennent malléables sans qu'ils ne se putréfient. Grâce à cette opération, les genitalia deviennent plus facilement extractibles car les tissus abdominaux sont assouplis.

\section{Montage des abeilles}

Un montage rigoureux des insectes permet de réaliser une collection pour l'inventaire et la détermination. Il est notamment judicieux de monter conventionnellement quelques spécimens afin de constituer une collection de référence, servant de base de travail. Pour la détermination, il est nécessaire de pouvoir visualiser certaines parties du spécimen. Ces parties sont plus ou moins importantes selon les genres concernés. En général il est important, parfois indispensable, de pouvoir observer :

- les cellules alaires antérieures, parfois postérieures;

- les pattes postérieures, parfois les antérieures ou les médianes, parfois encore les derniers articles (tarses, métatarses, griffes);

- les tergites (segments de la face dorsale de l'abdomen) et sternites (segments ventraux de l'abdomen);

- les différentes parties du thorax (pronotum, mesonotum, scutellum, postscutellum, propodeum);

- $\quad$ les articles antennaires (couleur, taille, nombre pour les sexes);

- $\quad$ les genitalia des mâles;

- la pilosité (densité et couleur);

- les mandibules et la langue dans certains cas (galea, glossa, palpes labiaux et maxillaires) surtout pour Andrena, Hylaeus, Nomada, Epeolus, Colletes;

Pour permettre une bonne observation, voici quelques conventions (Figure 3):

- L'épingle doit être plantée dans le thorax, latéralement pour préserver un des deux côtés. Afin d'uniformiser les hauteurs d'épinglage, on utilise un "bloc à piquer". L'insecte doit être disposé à $10 \mathrm{~mm}$ sous la tête de l'épingle tandis que les étiquettes sont disposées à 12, 16 et 20 mm de la pointe;

- Les pattes antérieures sont légèrement dirigées vers l'avant;

- Les pattes médianes et postérieures sont légèrement dirigées vers l'arrière;

- Les ailes sont étalées idéalement en delta (un seul côté peut suffire, le droit généralement); mais on peut aussi monter un côté avec les ailes accrochées (par les hamuli) et l'autre paire d'ailes décrochées. Les 2 paires d'ailes complètement ouvertes sont toutefois plus esthétiques. D'autre part, en tirant une paire

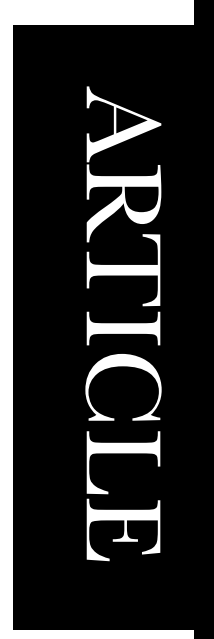



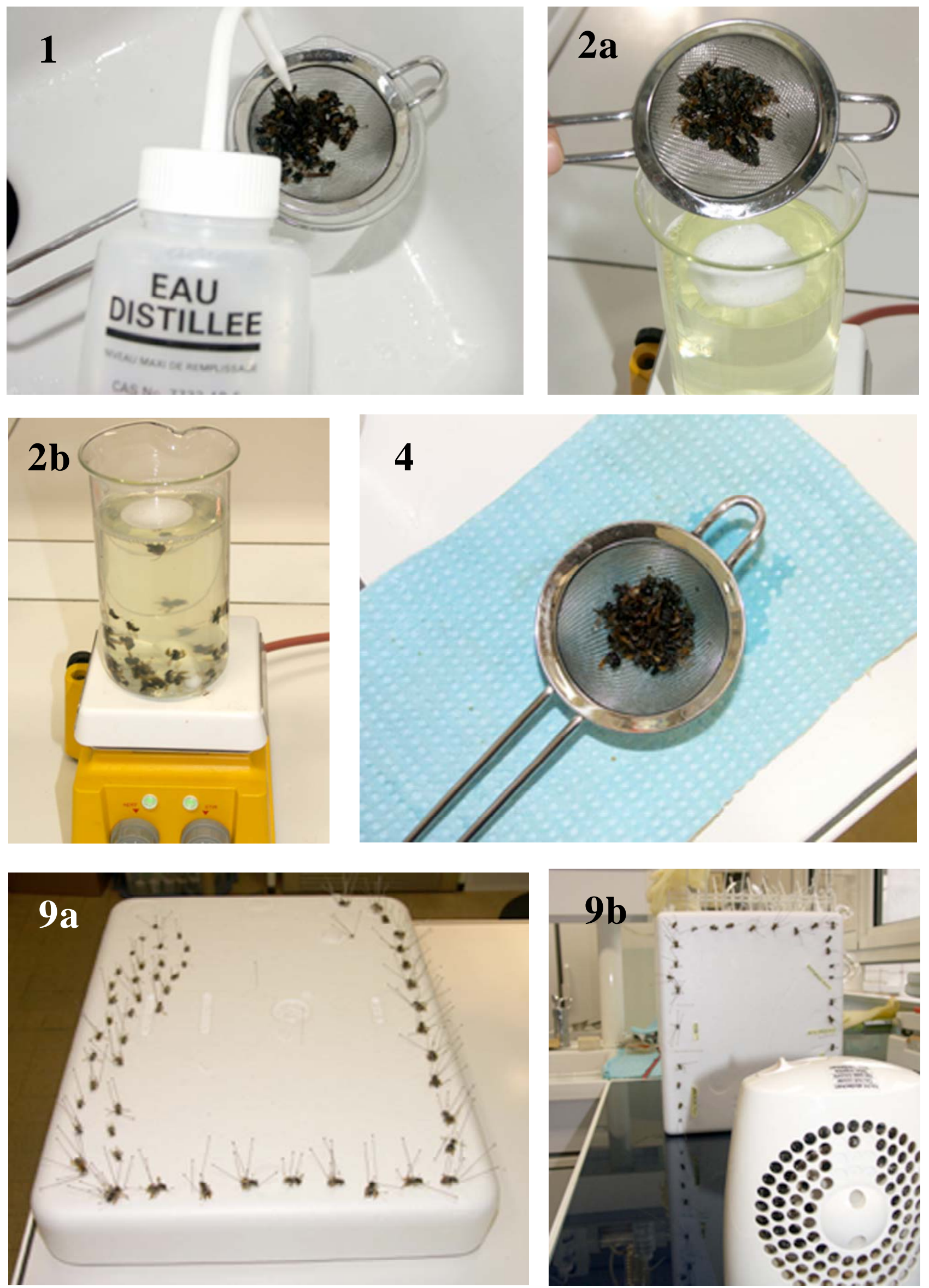

Figure 2. Principales étapes pour la préparation des spécimens d'abeilles conservées dans l'alcool; les numéros indiquent les différentes étapes et font référence au texte 
d'ailes, la seconde bouge parfois parallèlement; La langue est sortie et déployée, présentant si possible ses différentes parties (pas toujours utile pour la détermination);

- Les mandibules sont ouvertes (important pour voir le bord du clypeus);

- Les genitalia des mâles sont exposées ; si les édéages sont séparés de l'insecte, on peut les encoller sur une pastille cartonnée qui sera épinglée sous l'animal ; la face ventrale des genitalia doit être apparente. On peut en outre coller les parties génitales, ainsi que les sternites 7 et 8 (qui se détachent souvent avec), dirigés vers le haut;

- Les épingles d'usage le plus courant sont les $\mathrm{n}^{\circ}$ 3-1, 0, 00 ou des minuties selon la taille du spécimen ; un diamètre d'épingle trop gros abîme le mésonotum, un diamètre trop petit peut vibrer et casser à la manipulation;

- Un brossage des poils des abeilles peut s'avérer également utile et esthétique pour les spécimens d'une collection de référence;

- Les abeilles les plus petites (Ceratina, Nomioides, certains Lasioglossum...) sont montées sur minutie afin de limiter la perforation du thorax et de ne pas risquer de casser la bête (voir Figure 3-5). Pour cela, on plante la minutie dans le thorax de l'insecte, puis on plante la minutie avec l'insecte dans une bande d'émalène $(1,5 \mathrm{~cm})$ qui reçoit l'épingle "classique" pour l'étiquetage.

Pour les spécimens capturés au filet, les tissus sèchent naturellement. La raideur cadavérique a tendance à replier les articles, les pattes ou les ailes. Selon la taille des spécimens, on les monte le lendemain, parfois plusieurs jours après, et les appendices se mettent alors plus facilement en place. Pour les abeilles, il faut entre 1 et 5 jours pour que le montage soit aisé. On peut également les laisser au congélateur pendant quelques jours après la capture, ce qui empêche le spécimen de devenir trop sec. Si malgré tout l'insecte devient trop sec et cassant, il peut alors être ré-humidifié. Pour cela, on le place dans une boite hermétique avec un fond de coton imbibé d'eau pendant quelques jours, en surveillant qu'il ne se développe pas de moisissures.

Pour maintenir convenablement les abeilles le temps du séchage, on utilise des épingles qui calent les pattes, le corps et la tête. Les ailes sont disposées idéalement sur de fines "cales" d'émalène et maintenues par une bande de papier glace. A défaut, on peut utiliser des minuties et transpercer le bord d'attaque des ailes antérieures. Dans ce cas, les ailes de l'insecte sont extrêmement fragiles le temps du séchage et des précautions sont à prendre pour retirer les épingles et éviter de les déchirer.

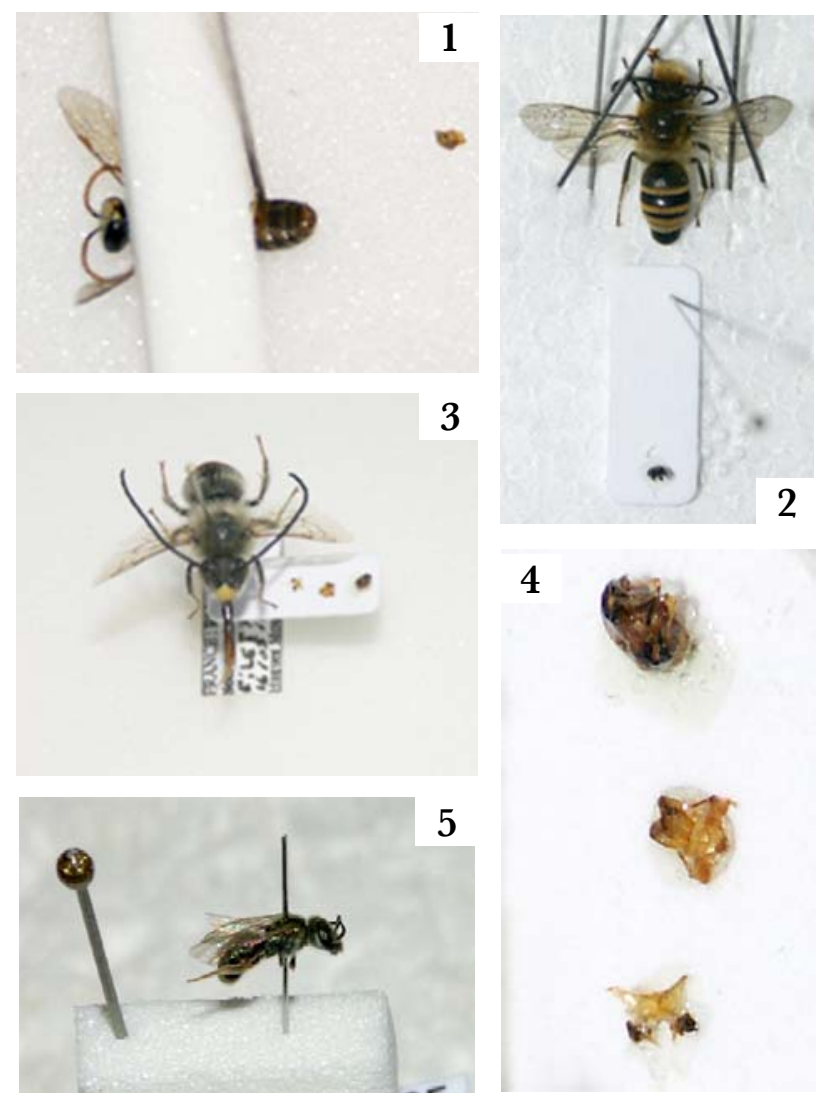

Figure 3. Extraction et montage des genitalia d'abeille. 1. Mise en place du spécimen pour l'extraction ; 2. préparation d'un spécimen pendant le séchage et le collage des genitalia sur paillette ; 3. un exemple de spécimen épinglé avec ses genitalia sur paillette ; 4. un agrandissement de la préparation (avec ici la séparation entre genitalia et les deux sternites terminaux dans le cas des Eucerini); 5. un montage sur minutie

\section{L'étiquetage}

L'objectif est d'avoir sur chaque spécimen toutes les informations essentielles. Il faut donc qu'elles puissent tenir sur une ou plusieurs étiquettes épinglées sous le spécimen. Il est important d'éviter de faire références à une autre source (base de données, cahier de capture...), car en cas de perte de ce document, toutes les informations essentielles liées aux spécimens seront également perdues. Mais cela n'empêche pas de consigner des observations sur un carnet de terrain ou des fichiers informatiques.

Les étiquettes sont rectangulaires et présentent chacune quatre lignes au plus (exceptionnellement cinq) arrangées de façon à ce que chaque ligne soit à peu près de même taille. On peut utiliser des abréviations si elles sont parfaitement explicites.

\section{Etiquette supérieure (Figure 4-1)}

L'étiquette supérieure reprend généralement la localité, la date de collecte et le nom du collecteur.

1. La ligne 1 de localité comprend les informations de la plus large à la plus locale (pays, département, commune); 
A cette information générale de localité, on peut ajouter une précision comme le nom du lieu-dit et également la distance et la direction de la ville la plus proche si c'est utile;

2. La ligne 2 comprend la date. Les mois ne sont généralement pas écrits en chiffres décimaux mais en chiffres romains ou en lettres (entièrement ou abrégés). Cette règle permet d'éviter les confusions avec les dates anglaises ou américaines qui placent l'indication du mois avant le jour. Il convient également de noter l'année entièrement pour éviter les confusions dues aux changements de siècles;

3. La ligne 3 porte le nom du ou des collecteurs (écrit entièrement).

\section{Deuxième étiquette (Figure 4-2)}

Elle contient d'autres informations notamment climatiques et écologiques (qui peuvent s'avérer d'une grande importance):

1. Les coordonnées géographiques données par GPS (Lat-long WGS84) ou dans le système de coordonnées locales (par exemple Lambert 3 pour le Sud de la France);

2. L'espèce florale sur laquelle a été capturé le spécimen, avec de préférence le nom scientifique. Cette indication est particulièrement intéressante pour obtenir des informations sur les relations entre plantes et insectes;

3. Des informations concernant l'habitat dans lequel s'est effectuée la capture. Exemple : talus forestier, haie composite...

4. La méthode de collecte: filet, coupelle colorée, etc.;

5. La période de la journée et d'autres informations portant sur les données climatiques.

\section{Etiquette inférieure (Figure 4-3)}

L'étiquette de détermination est généralement placée tout en dessous et contient :

- le nom latin actuel;

- le sexe;

- si possible l'indication du sous-genre;

- le nom du parrain et l'année de description;

- enfin, le nom du déterminateur précédé par

"det." ainsi que la date de détermination;

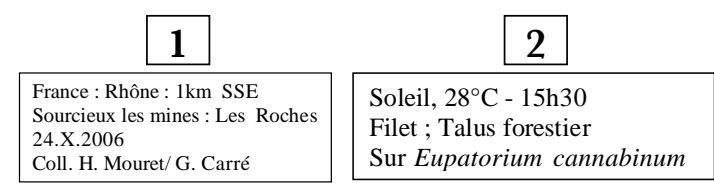

\begin{tabular}{|l|}
\multicolumn{1}{|c|}{$\mathbf{3}$} \\
\hline Halictus scabiosae 9 \\
(Rossi, 1790) \\
det. A. Pauly 2006 \\
\hline
\end{tabular}

Figure 4. Exemple d'étiquettes. 1. Etiquette "de récolte"; 2. Etiquette "écologique"; 3. Etiquette "taxonomique"

\section{Ecritures, impressions}

Les encres subissent les outrages du temps, notamment à cause des UV et une fois dégradées, les données sont perdues à jamais. Il convient donc de choisir sérieusement les modalités d'écriture ou d'impression.

Concernant les impressions, les avis sont partagés. Certains préconisent l'utilisation d'imprimantes laser ou, à défaut de photocopier ses impressions, car les encres tiendraient mieux dans le temps ; tandis que d'autres prétendent que les imprimantes à jet d'encre sont plus fiables sur la durée.

L'encre de Chine reste une excellente option car elle résiste pendant des siècles.

\section{Réalisation et pérennité d'une collection}

\section{Réalisation d'une collection}

L'homogénéité dans le choix du matériel (boîtes, étiquettes, épingles, montage des spécimens, écritures...) est un gage de facilité d'usage et de cohérence dans une collection. Chacun pourra faire ses propres choix en fonction de ses affinités, mais il reste important de s'y tenir.

1. Quelles boîtes utiliser? Il existe des boîtes "standard", de type Muséum ; il s'agit de boîtes en bois, dont la surface supérieure est vitrée (ou pas) et les bordures brutes ou recouverte de papier collant (vert et marron, noir...). Les tailles varient de $40 \times 50 \mathrm{~cm}$, $30 \mathrm{x} 40 \mathrm{~cm}$ ou $20 \times 10 \mathrm{~cm}$. Les boîtes vitrées permettent un contrôle visuel plus facile, mais sont plus lourdes, plus chères et plus fragiles. Selon la collection qu'on envisage et la séparation des spécimens (en familles, tribus...), on utilisera les boîtes qui conviennent.

\section{Disposition des spécimens dans les boîtes. Encore} pour des raisons d'esthétique, un placement linéaire propre rend les boîtes plus agréables à regarder. Mais pour des raisons pratiques, il convient que les spécimens ne se touchent pas, afin de ne pas les casser lors de manipulations; d'autre part la lecture des étiquettes s'en trouvera aussi facilitée. On devra prendre particulièrement garde aux appendices qui pourraient 
dépasser (antennes, pattes) et qui sont encore plus fragiles. On peut utiliser un fil de couture tendu entre 2 épingles pour se donner l'horizontale ou la verticale. En outre, les boîtes sont organisées selon les différents genres (sous-genres et tribus) et les espèces placées par ordre systématique (phylogénie) de préférence ; certains utilisent un ordre alphabétique, scientifiquement moins rigoureux. Sans tomber dans l'excès, il est nécessaire d'avoir plusieurs exemplaires d'une même espèce et de chaque sexe pour se faire une idée de la variabilité de certains critères systématiques.

\section{Conservation et protection des collections}

Comme toute collection d'insectes, une collection d'abeilles doit être surveillée, car elle peut subir diverses agressions (Figure 5) :

- attaques d'insectes nécrophages, comme les Dermestidae ;

- moisissures et autres champignons qui se développent sur la matière organique en milieu trop humide.

Les produits utilisés par les entomologistes dans les décennies précédentes sont extrêmement nocifs pour l'homme notamment en cas de contacts (respiration) prolongés ou fréquents. C'est le cas de la créosote de hêtre, de la naphtaline, du paradichlorobenzène, du lindane... Il existe néanmoins des méthodes plus douces mais efficaces pour stériliser les boîtes de collection et pour prévenir l'arrivée de Dermestidae :

1. Les huiles essentielles (thym, romarin...) sont utilisées pour repousser certains insectes gênants. Sont-elles efficaces contre les dermestes et autres mangeurs de collection? Nous n'avons pas d'informations précises à ce sujet, mais en tout cas, elles ont l'avantage de sentir bon. Plusieurs de nos boîtes sont conservées depuis quelques années avec des huiles essentielles, aussi nous envisageons de tester sérieusement leur efficacité.

2. La congélation. Une bonne solution consiste à se procurer un grand congélateur. Les dermestes ne résistent pas à des températures de $-18^{\circ} \mathrm{C}$, mais il se peut que leurs œufs le puissent... Le protocole consiste donc à placer au congélateur les boîtes dans un sac plastique (pour éviter la condensation) pendant une dizaine de jours, que l'on répète à 10 jours d'intervalle (temps d'incubation des œufs). On peut ainsi largement faire tourner toutes les boîtes d'une collection tout au long de l'année.

Enfin, pour conserver les collections sur le long terme, il faut :

1. Contrôler régulièrement les boîtes (au moins 2 fois par an) et prévenir toute attaque ;

2. Conserver sa collection dans un milieu frais $\left(15-20^{\circ}\right.$ C) et $\sec (<50 \%$ d'hygrométrie); la température optimale de conservation restant celle d'un réfrigérateur (environ $4-6^{\circ} \mathrm{C}$ ) ;
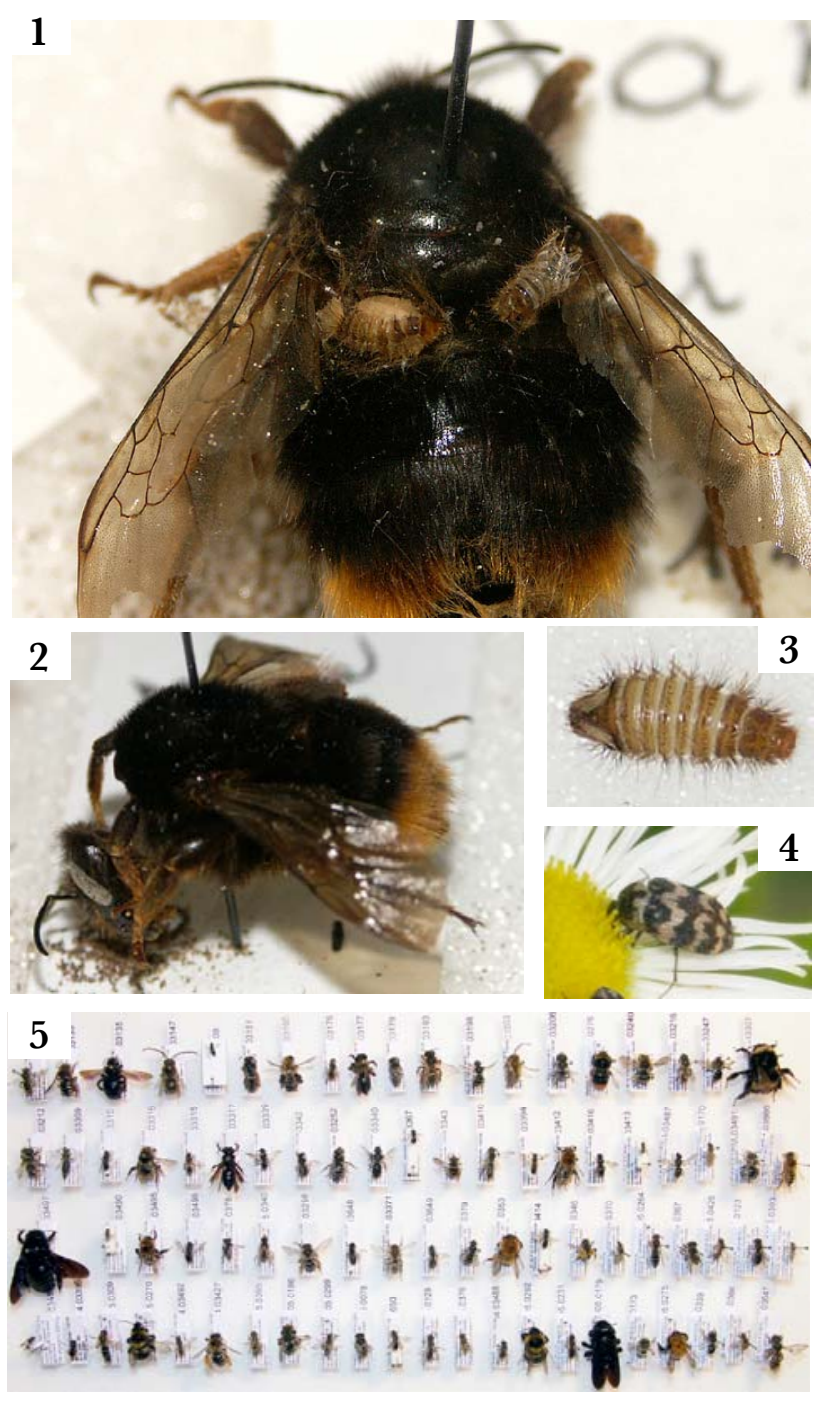

Figure 5. Protection des collections entomologiques. 1 et 2: Dégâts sur les spécimens d'une collection engendrés par l'attaque de dermestes; 3 et 4: photos de la larve de dermeste (3) et d'un dermeste adulte (4); 5: exemple de boite avec plusieurs spécimens.

3. Un passage régulier au congélateur pour tuer les éventuels envahisseurs (deux fois 10 jours à $-18^{\circ} \mathrm{C}$ à 10 jours d'intervalle).

\section{Envois de spécimens}

Pour le transport des spécimens montés, on se procure des petites boîtes cartonnées solides, dont le fond sera recouvert d'une plaque d'émalène. Il est nécessaire d'indiquer que le colis est fragile. Habituellement la mention "insectes naturalisés morts; usage scientifique" permet d'éviter toute ouverture intempestive. On peut également utiliser de petites boîtes en bois (plus solides, mais plus lourdes) qui seront convenablement emballées (papier à bulles, polystyrène, journaux en boule...) et calées dans un carton adéquat. A l'intérieur des boîtes, les spécimens doivent être précautionneusement séparés et calés à l'aide d'épingles, afin d'éviter qu'ils ne tournent et se cassent; en particulier les grosses espèces dont l'abdomen est lourd. Certains préconisent encore d'épingler un petit morceau de coton dans un coin de la boîte, afin de "récupérer" les spécimens ou les articles baladeurs. 
Une autre précaution consiste enfin à favoriser les transports directs (déplacements de personnes), plutôt que les services d'envois (poste ou transporteur), dont les risques ne sont pas toujours maîtrisés.

\section{Conclusion}

Une collection de référence reste nécessaire, indispensable même, pour l'entomologiste qui désire approfondir l'étude des insectes.

Dans le cadre d'ALARM, nous avons donc dû entreprendre la construction d'une collection de référence pour approfondir l'étude des abeilles. Voici donc rassemblées ici quelques règles qui nous ont semblé utiles pour commencer. Bien entendu, il n'y a aucune obligation d'appliquer à la lettre toutes ces recommandations. Elles peuvent être adaptées en fonction des besoins et contraintes de chacun. Considérons que les experts du monde entier seront toujours nettement mieux disposés pour examiner vos spécimens si ceux-ci sont bien conservés et bien montés...

\section{Références bibliographiques}

Buchmann SL \& Nabhan GP, 1996. The forgotten Pollinators. Island Press, Washingt on, D.C. Shearwater Books, Coverlo, California, 320p.
Costanza R, d'Arge R, Groot R de, Farber S, Grasso M, Hammon B, Limburd K, Naeem S, O'Neill RV, Paruelo J, Raskin RG, Sutton P \& Belt M van den, 1997. The value of the world's ecosystem services and natural capital. Nature 387: 253-259.

Kremen C, Williams NM \& Thorp RW, 2002. Crop pollination from native bees at risk from agricultural intensifications. Proc. Natl. Acad. Sci. 99: 16812-16816.

Kremen G, Williams NM, Bugg RL, Fay JP \& Thorp RW, 2004. The area requirements of ecosystem service: crop pollination by native bee communities in California. Ecol. Lett. 7: 1109-1119.

Michener GD, 2000. The bees of the world. Johns Hopkins University Press, Baltimore USA

Rasmont P, Ebmer PA, Banaszak J \& van der Zanden G, 1995. Liste taxonomique des abeilles de France, de Belgique, de Suisse et du Grand-Duché de Luxembourg. Bull. Soc. Entomol. France 1100 (HS): 1-98.

Settele J, Hammen V, Hulme P, karlson U, Klotz S, Kotarac M, Kunin W, Marion G, O'Connor M, Petanidou T, Peterson K, Potts S, pritchard H, Pysek P, Rounsevell M, Spangenberg J, Steffan-Dewenter I, Sykes M, Vighi M, Zobel M \& Kühn I. 2005. ALARM: Assessing large-scale environmental risks for biodiversity with tested methods. GAIA $14: 69-72$.

Stuart Chapin FS, Zavaleta ES, Eviner VT, Naylor RL, Vitousek PM, Reynolds HL, Hooper DU, Lavorel S, Sala OE, Hobbie SE, Mack MG \& Díaz S, 2000. Consequences of changing biodiversity. Nature 405: 234 - 242.

Williams IH, 1994. The dependence of crop pollination within the European Union on pollination by honey bees. Agricultural Zoology Reviews 6: 229-257.

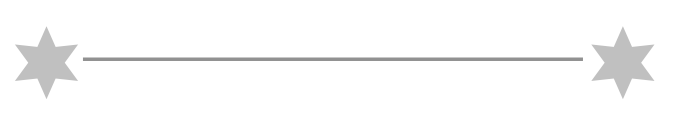

